\title{
Esposizione a fumo di tabacco e malattie respiratorie infantili
}

\section{Tobacco smoke esposure and respiratory disease in children}

\author{
Velia Malizia
}

\section{Riassunto}

Negli ultimi 30 anni sono state prodotte prove certe circa le conseguenze dannose dell'esposizione ambientale al fumo di tabacco. II fumo di seconda mano (second hand smoke - SHS) o fumo di tabacco "ambientale" (environmental tobacco smoke - ETS) costituisce la principale fonte di inquinamento atmosferico interno. Esistono prove convincenti che suggeriscono una relazione causale tra esposizione a SHS e incidenza di malattie respiratorie nei bambini. È noto anche che il fumo di tabacco materno durante la gravidanza aumenta il rischio di respiro sibilante, asma e ridotta funzionalità polmonare nella prole, indipendentemente dall'esposizione al fumo durante la vita postnatale. Recenti studi hanno dimostrato, inoltre, come l'esposizione in casa al fumo di tabacco di terza mano (THS) possa danneggiare le vie respiratorie inferiori dei bambini. L'uso delle sigarette elettroniche, notevolmente aumentato negli ultimi 5 anni, è una nuova ulteriore minaccia per la salute dei bambini e degli adolescenti. La conoscenza degli stretti legami esistenti tra l'esposizione al fumo passivo domestico e lo sviluppo delle malattie respiratorie infantili non può che essere incoraggiata, al fine di contribuire alla tutela della salute di tali soggetti fragili.

Parole chiave: inquinamento ambientale, fumo di tabacco, malattie respiratorie, asma, bambino.

\begin{abstract}
Over the last 30 years certain proof has been produced about the dangerous consequences of exposure to tobacco smoke. Cigarette smoke remains the most common indoor polluter [11] Second Hand Smoke (SHS) or Environmental Tobacco Smoke (ETS) constitute the principal font of indoor pollution. Convincing proof exists which suggests a reasonable relationship between exposure to SHS and respiratory diseases in children. It is also known that maternal smoking during pregnancy increases the risk of wheezing, asthma and a reduced lung function in the child, independently of the exposure to smoking after birth. All over the world, in the last 5 years the use of the electronic cigarette has increased remarkably. It is a new additional threat to the health of children and adolescents. Knowledge of the close links between exposure to domestic passive smoke and the development of childhood respiratory diseases can only be encouraged, in order to contribute to the protection of the health of these fragile individuals.
\end{abstract}

Key words: environmental pollution, tobacco smoking, respiratory diseases, asthma, child. 


\section{Introduzione}

L'Organizzazione Mondiale della Sanità (OMS) ha evidenziato che lo stile di vita dei Paesi occidentali economicamente più ricchi è associato a un aumento delle malattie croniche non trasmissibili [1]. La crescente urbanizzazione, lo sviluppo industriale e la rapida crescita della popolazione hanno contribuito a significativi cambiamenti dello stile di vita (dieta, tempo trascorso a casa, attività fisica) con maggior rischio di esordio e prevalenza di malattie respiratorie [2-5]. I bambini sono più vulnerabili degli adulti ai rischi ambientali soprattutto perché organi e apparati (respiratorio, immunitario, nervoso centrale, digerente) non sono ancora completamente sviluppati; inoltre, a causa delle dimensioni dei bronchi i tassi di inalazione di inquinanti aerei sono superiori a quelli degli adulti [1]. Nei Paesi industrializzati, il 90\% delle persone trascorre il suo tempo in ambienti chiusi, dove il numero e le concentrazioni di inquinanti possono essere superiori a quelli presenti negli spazi aperti $[6,7]$. L'inquinamento dell'aria all'interno delle case è costituito da una complessa miscela di agenti sia provenienti dall'ambiente esterno che generati da sorgenti interne [8]. Per ambienti indoor si intendono gli ambienti confinati di vita e di lavoro non industriali (per quelli industriali vige una specifica normativa), e in particolare quelli adibiti a dimora, svago, lavoro e trasporto [Accordo del 27/09/2001 tra il Ministero della Salute, le Regioni e le Province autonome]. Le sostanze in grado di alterare la qualità dell'aria indoor sono agenti chimici, fisici e biologici. Tra le fonti di inquinanti interni più comuni troviamo: fumo di tabacco; processi di combustione (stufe a pellet, camini); prodotti per la pulizia e la manutenzione della casa, antiparassitari, solventi, etc.; inquinanti derivanti dall'utilizzo di strumenti di lavoro quali stampanti, plotter e fotocopiatrici, nonché prodotti per l'hobbistica (per esempio, colle e vernici); malfunzionamento di impianti (condizionatori, umidificatori, impianti idraulici); polvere di casa (ricettacolo per i microrganismi) e inquinanti provenienti dall'aria esterna [9]. L'inquinamento dell'aria indoor rappresenta un rischio per la salute umana, in particolare per i bambini e per i soggetti con malattie respiratorie [9]: esso è stato responsabile, nel 2016, di oltre 400.000 decessi di bambini di età inferiore a 5 anni [10].

\section{Esposizione al fumo di tabacco e malattie respiratorie nei bambini}

Negli ultimi 30 anni sono state prodotte prove certe circa le conseguenze dannose dell'esposizione ambientale a fumo di tabacco. II fumo di seconda mano (second hand smoke - SHS) o fumo di tabacco "ambientale" (environmental tobacco smoke - ETS) costituisce la principale fonte di inquinamento indoor e contiene più di 4.500 sostanze chimiche, alcune delle quali dotate di proprietà irritanti (per esempio, nicotina) e altre riconosciute come cancerogene (per esempio, 4-aminobifenile, benzopirene, toluene, dimetil-nitrosamina) $[11,12]$. L'esposizione al fumo di sigaretta altera i meccanismi immunitari coinvolti in malattie allergiche e compromette le risposte dell'epitelio bronchiale modificando l'espressione e l'attivazione dei recettori dell'immunità innata, determina la distruzione dei desmosomi e delle tight junction con aumento della permeabilità agli allergeni, inquinanti e agenti infettanti e rilascio dei mediatori della infiammazione (IL-8, IL-1 $\beta$, sICAM-1) [13]. L'interazione gene-ambiente condiziona nei bambini la suscettibilità a sviluppare malattie respiratorie dovute alla esposizione passiva al fumo, durante la vita pre e postnatale. Per esempio, in bambini con genotipo $(\mathrm{M}+)$ della glutatione transferasi (GSTM1+) esposti in utero al fumo materno in gravidanza non vi è associazione con asma o wheezing, mentre in bambini con genotipo (GSTM1-) si osserva una maggiore prevalenza di asma a insorgenza precoce, asma corrente, asma persistente $[14,15]$.

\section{SHS e malattie respiratorie nei bambini}

Esistono prove convincenti a supporto della relazione causale tra esposizione a SHS e incidenza di malattie respiratorie nei bambini $[16,17]$. L'esposizione a fumo materno pre o postnatale è stata associata a un aumento significativo di rischio di respiro sibilante (28$70 \%$ ) e di asma fino a 18 anni (2085\%) [18]. Anche in assenza di esposizione a fumo materno, l'esposizione a SHS nell'infanzia aumenta il rischio di eczema con sensibilizzazione allergica fino a 16 anni $[19,20]$. Per le patologie delle basse vie aeree l'esposizione passiva al fumo dei genitori è un fattore di rischio per tosse, catarro, sibili e asma [18,21]. Nei bambini di età compresa tra i 18 mesi e i 5 anni, conviventi con madri fumatrici, è significativamente aumentata la sintomatologia respiratoria [22]. Una metanalisi condotta in soggetti di età compresa tra i 6 e i 18 anni ha evidenziato che la durata di esposizione a SHS è il più importante fattore di rischio per l'esordio di patologie respiratorie. In particolare, nei bambini in età scolare sono riportati i seguenti rischi relativi per asma in vita (RR 1,48; $95 \% \mathrm{Cl}: 1,32-1,65)$, asma corrente (RR 1,25; 95\% Cl: 1,21-1,30) ed esordio di asma (RR 1,21; $95 \% \mathrm{Cl}$ : 1,08-1,36) [23]. In uno studio trasversale condotto su un campione di 2.150 adolescenti di età 10-17 anni è stata dimostrata un'associazione significativa tra l'esposizione a fumo passivo o muffa/umidità e maggior rischio di rinocongiuntivite [24]. In tale studio vengono fornite anche le stime del rischio attribuibile di popolazione, in base alle quali il $18,1 \%$ dei casi di asma e il $18,8 \%$ dei casi di rinocongiuntivite potrebbero essere prevenuti eliminando l'esposizione al fumo passivo [24]. Lo studio italiano sui disturbi respiratori nell'infanzia e 
I'ambiente (SIDRIA 2) condotto su bambini e adolescenti italiani ha valutato l'associazione tra la prevalenza di tre disturbi respiratori (asma corrente, sintomi asmatici, tosse o catarro persistente) e l'esposizione al fumo passivo. Lo studio ha valutato separatamente il contributo del fumo della madre, del padre e di entrambi i genitori e il numero di sigarette fumate. La presenza di almeno un genitore fumatore è risultata associata a un maggior rischio di disturbi asmatici e in entrambe le fasce di età i rischi aumentano particolarmente al crescere del numero di sigarette fumate dalla madre [25].

\section{THS e malattie respiratorie nei bambini}

Un altro aspetto è quello relativo all'inquinamento del fumo di tabacco cosiddetto di terza mano (THS), costituito da sostanze inquinanti che rimangono sulle superfici degli oggetti e nella polvere di casa. Queste sostanze vengono successivamente rilasciate e/o rimesse in circolo sotto forma gassosa, reagendo poi con altri composti che si trovano nell'ambiente per formare inquinanti secondari $[26,27]$. In particolare, mentre l'esposizione a SHS deriva da inalazione involontaria del fumo che si libera dalla combustione della sigaretta nel flusso principale (main-stream) e nel flusso laterale (side-stream), l'esposizione a THS è derivata dall'inalazione, ingestione $\mathrm{O}$ assorbimento cutaneo involontario di sostanze inquinanti generate dal fumo di sigarette presenti sulle superfici e nella polvere di casa. Gli inquinanti del THS possono persistere negli ambienti interni anche per diversi giorni o mesi dopo che il tabacco è stato fumato e possono accumularsi sulle superfici, diventando progressivamente più tossici $[26,27]$. Molti studi hanno dimostrato che il fumo di SHS ha gravi effetti sulla salute dei bambini $[28,29]$; pochi invece sono gli studi condotti in bambini sulla esposizione a THS. Un recente studio con- dotto da Jung e coll. [30] ha dimostrato che l'esposizione in casa a THS può aumentare nei bambini il rischio di sintomi come la tosse, anche se in misura minore rispetto al fumo SHS. Le limitate prove disponibili fino a oggi non consentono di stimare il ruolo di THS nel causare problemi di salute nelle persone esposte, pertanto sono necessarie ulteriori indagini per studiare gli effetti THS sulle malattie respiratorie relativamente alle diverse vie di esposizione (inalazione, contatto cutaneo, ingestione di polvere) e ai tempi di esposizione (brevi e lungo termine).

\section{Sigarette elettroniche e malattie respiratorie nei bambini}

Negli ultimi 5 anni in tutto il mondo l'uso di sigarette elettroniche è notevolmente aumentato $[31,32]$. I livelli di sostanze tossiche nel vapore delle sigarette elettroniche sono inferiori a quelli del fumo di tabacco [33], ma i dati sulla sicurezza relativi al loro utilizzo sono limitati. Alcuni dati riportano che il vapore della sigaretta elettronica può produrre stress ossidativo e infiammazione nelle vie aeree e può esacerbare $\mathrm{o}$ indurre rinite, asma, eczema e sintomi allergici [34-36]. La sigaretta elettronica rappresenta una nuova minaccia per la salute di bambini e adolescenti. Non vi sono ancora norme che regolamentano i contenuti e quantità delle sostanze presenti nella sigaretta elettronica. Questi dispositivi contengono composti organici (glicerolo propanidiolo), nicotina e aromi. Gli aromi aumentano l'attrattività dei giovani per questo tipo di prodotto e ne espandono il marketing $[37,38]$. I produttori di sigarette elettroniche utilizzano strategie diverse e creative per aumentarne la vendita, nonostante i diffusi divieti della vendita di questi prodotti a persone di età inferiore ai 18 anni. Le sostanze presenti nella sigaretta elettronica non sono inerti e hanno dimostrato in vitro di danneggiare le cellule epiteliali delle vie aeree [39]. Studi sugli adolescenti, condotti negli Stati Uniti, Corea del Sud e Cina, hanno collegato il loro utilizzo a sintomi respiratori cronici o ricorrenti $[40,41]$. Negli ultimi dieci anni, la diffusione dell'uso delle sigarette elettroniche è aumentata rapidamente tra i giovani di molti Paesi. Un'indagine condotta negli Stati Uniti, Canada, Regno Unito e Australia, tra i giovani fumatori e tra i fumatori saltuari, ha evidenziato che l'elevato uso delle sigarette elettroniche è motivato dalla percezione che siano meno dannose rispetto alle sigarette tradizionali [42]. In tal senso, gli adolescenti che usano e-cig tendono a essere quelli a più alto rischio per inizio della dipendenza tabagica $[43,44]$. Infatti, è stato riportato che l'uso di sigarette elettroniche negli adolescenti non diminuisce la probabilità di iniziare a fumare sigarette tradizionali; piuttosto, tra i giovani, I'uso della sigaretta elettronica, lo "svapo", è associato a quello dell'uso della sigaretta tradizionale portando così a un aumento del numero di fumatori duali, che quindi fumano contemporaneamente sigaretta elettronica e sigaretta tradizionale [45].

\section{Fumo materno e malattie respiratorie nel bambino}

L'esposizione al fumo di tabacco materno durante la gravidanza aumenta il rischio di respiro sibilante, asma e ridotta funzionalità polmonare nei bambini, indipendentemente dall'esposizione al fumo durante la vita postnatale. Gli effetti diretti del fumo materno in gravidanza sullo sviluppo polmonare sono principalmente dovuti ai componenti della combustione del tabacco trasferiti al feto attraverso la placenta. La nicotina è in grado di attraversare la placenta e il suo metabolita "cotinina" si accumula nel liquido amniotico e in diversi tessuti di organi fetali (inclusi i polmoni). Pertanto, il feto è esposto a livelli ancora più elevati di nicotina rispetto a quelli della madre fuma- 
trice [46]. In 10.860 partecipanti di cinque studi di coorte di nascita europei (progetto MeDALL) è stata valutata I'associazione tra l'esposizione al fumo materno durante la gravidanza e patologie respiratorie durante l'infanzia [47]. II fumo materno durante la gravidanza $(\geq 10$ sig/die) è risultato associato ad asma a esordio precoce [OR 2,07; $95 \% \mathrm{Cl}: 1,60-2,68)$, asma persistente [OR 1,66; $95 \% \mathrm{Cl}$ : 1,29$2,15)$ e rinocongiuntivite persistente [OR 1,55; 95\% Cl: 1,09-2,20) [47]. Una revisione sistematica di 43 articoli ha evidenziato che l'esposizione al fumo materno in gravidanza si associa a un aumento del rischio di respiro sibilante in bambini < 6 anni (OR 1,36; 95\% $\mathrm{Cl}: 1,19-1,55)$ e di respiro sibilante o asma nei bambini di 6 anni (OR 1,22; 95\% Cl: 1,03-1,44) [48]. Carroll e coll. hanno riportato che, nel primo anno di vita, i bambini nati da madri asmatiche fumatrici in gravidanza hanno circa il $50 \%$ di rischio in più di andare incontro a bronchiolite, rispetto a bambini nati da madri non fumatrici in gravidanza [49]. Questi dati sottolineano la necessità di applicare estensivamente e precocemente interventi di prevenzione nella popolazione vulnerabile dei bambini e degli adolescenti.

\section{Prevenzione e monitoraggio}

La diffusa consapevolezza dei danni causati da esposizione a inquinamento indoor ha portato all'attivazione in oltre la metà dei Paesi europei di piani di azione nazionali sulla salute [50]. La ricerca scientifica ha evidenziato l'importanza di favorire la diffusione delle conoscenze sui danni alla salute provocati dall'esposizione al fumo passivo e ha sollecitato misure di protezione dal fumo passivo in ambienti aperti e confinati. L'importanza degli effetti di un programma di intervento formativo sui genitori relativo ai fattori di rischio per asma, incluso l'ETS, è evidente nello studio di Gerald e coll. [51]. Gli autori riportano che nel cam- pione di bambini con asma persistente i cui genitori avevano ricevuto il supporto informativo sui rischi da esposizione a fumo passivo vi era un minor numero di ricoveri $(p=0,034)$ e di visite al Pronto Soccorso (DE) $(p<0,001)$ insieme a una maggiore cessazione tabagica nei genitori, rispetto a un gruppo di controllo non informato. Una revisione sistematica di Nurmagambetov e coll. ha valutato l'efficacia degli interventi ambientali domiciliari sulla riduzione della morbilità dell'asma [52]. Lo studio ha evidenziato che gli interventi mirati alla riduzione dell'esposizione ai fattori scatenanti l'asma (allergeni e irritanti) mediante il controllo ambientale (valutazione del domicilio, comunicazione-educazione, interventi di recupero) possono migliorare gli indicatori del suo esito. Infine, in merito alla prevenzione dell'esposizione al fumo di tabacco GARD Italia (Alleanza globale contro le malattie respiratorie croniche) ha realizzato un documento sulla tutela dal fumo passivo negli spazi confinati o aperti non regolamentati dalla Legge $n$. 3/2003, art. 51 (legge Sirchia) e successive modificazioni. II documento riassume $\mathrm{i}$ dati relativi ai danni da fumo passivo, la normativa e le buone pratiche nazionali e internazionali relative al fumo in ambienti indoor e spazi aperti [53]. Specifiche attività informative e dedicati piani di azione devono essere promossi per contrastare il fumo passivo in ambiente domestico, dove il livello di esposizione raggiunge elevati valori causando effetti di morbilità acuta e cronica sulla salute respiratoria.

\section{Conclusioni}

Evitare l'esposizione agli inquinanti ambientali è particolarmente importante per la salute dei bambini. Revisioni sistematiche e metanalisi dimostrano che l'inquinamento dell'aria indoor aumenta il rischio di asma bronchiale e di sintomi correlati all'asma. La ricerca sui meccanismi alla base degli effetti nocivi dell'inquinamento atmosferico sulla salute ha suggerito di rafforzare e applicare una legislazione mirata al controllo dell'inquinamento dell'aria sia all'aperto sia al chiuso. Sebbene I'Unione Europea abbia sviluppato negli ultimi decenni un'ampia serie di direttive e linee di indirizzo che hanno portato indubbiamente a considerevoli miglioramenti della qualità dell'aria che respiriamo, i dati continuano a confermare che il carico di malattie legate all'inquinamento atmosferico tra la popolazione europea rimane ancora troppo elevato. La conoscenza degli stretti legami esistenti tra l'esposizione al fumo passivo domestico e lo sviluppo delle malattie respiratorie infantili non può che essere incoraggiata, al fine di contribuire alla tutela della salute di tali soggetti fragili.

[Tabaccologia 2021; XIX(3):27-32] https://doi.org/10.53127/tblg-2021-A022

\section{Velia Malizia \\ Istituto per la Ricerca e I'Innovazione Biomedica (IRIB), Consiglio Nazionale delle Ricerche, Palermo \\ $\bowtie$ velia.malizia@irib.cnr.it \\ - Disclosure: gli autori dichiarano I'assenza di conflitto d'interessi.}




\section{Bibliografia}

1. World Health Organization (WHO). Environment, climate change and health. Children's environmental health. 18 May 2020. http://www.who.int/ceh/risks/en/

2. Burbank AJ, Sood AK, Kesic MJ, Peden DB, Hernandez ML. Environmental determinants of allergy and asthma in early life. J Allergy Clin Immunol 2017;140:1-12. https://doi. org/10.1016/j.jaci.2017.05.010

3. Kanchongkittiphon W, Mendell MJ, Gaffin JM, Wang G, Phipatanakul W. Indoor environmental exposures and exacerbation of asthma: an update to the 2000 review by the Institute of Medicine. Environ Health Perspect 2015;123:620. https://doi.org/10.1289/ehp.1307922

4. Morgan BW, Siddharthan $T$, Grigsby MR, Pollard SL, Kalyesubula R, Wise RA, et al. Asthma and allergic disorders in Uganda: a population-based study across urban and rural settings. J Allergy Clin Immunol Pract 2018;6:1580-7. https://doi.org/10.1016/j.jaip.2017.11.032

5. Murrison LB, Brandt EB, Myers JB, Hershey GKK. Environmental exposures and mechanisms in allergy and asthma development. J Clin Invest 2019;129:1504-15. https:// doi.org/10.1172/JCl124612

6. Chen L, Hu G, Fan R, Lv Y, Dai Y, Xu Z. Association of PAHs and BTEX exposure with lung function and respiratory symptoms among a nonoccupational population near the coal chemical industry in Northern China. Environ Int 2018;120:480-8. https://doi.org/10.1016/j.envint.2018.08.004

7. Baloch RM, Maesano CN, Christoffersen J, Banerjee S, Gabriel M, Csobod É, et al.; SINPHONIE Study group. Indoor air pollution, physical and comfort parameters related to schoolchildren's health: data from the European SINPHONIE study. Sci Total Environ 2020;739:139870. https://doi. org/10.1016/j.scitotenv.2020.139870

8. Laumbach R, Meng Q, Kipen H. What can individuals do to reduce personal health risks from air pollution? J Thorac Dis 2015;7:96-107. https://doi.org/10.3978/j.issn.2072-1439. 2014.12.21

9. Lepore A, Ubaldi V, Brini S. Inquinamento indoor: aspetti generali e casi studio in Italia. Istituto Superiore per la Protezione e la Ricerca Ambientale (ISPRA) Roma, 2010.

10. World Health Organization (WHO). Burden of disease from ambient air pollution for 2016. Summary of results. Version 2 May 2018.

11. World Health Organization (WHO). WHO report on the global tobacco epidemic 2009: implementing smoke-free environments. World Health Organization Geneva, 2009.

12. Chan-Yeung M, Dimich-Ward H. Respiratory health effects of exposure to environmental tobacco smoke. Respirology 2003;8:131-9. https://doi.org/10.1046/j.1440-1843.2003.00453.x 13. Olivera DS, Boggs SE, Beenhouwer C, Aden J, Knall C, et al. Cellular mechanisms of mainstream cigarette smoke-induced lung epithelial tight junction permeability changes in vitro. Inhal Toxicol 2007;19:13-22. https://doi.org/10.1080/ 08958370600985768

14. Gilliland FD, Li YF, Dubeau L, Berhane K, Avol E, McConnell $R$, et al. Effects of glutathione S-transferase $\mathrm{MI}$, maternal smoking during pregnancy, and environmental tobacco smoke on asthma and wheezing in children. Am J Respir Crit Care Med 2002;166:457-63. https://doi.org/10.1164/rccm.2112064

15. Kabesch M, Hoefler C, Carr D, Leupold W, Weiland SK, von Mutius E. Glutathione S transferase deficiency and passive smoking increase childhood asthma. Thorax 2004;59:569-

73. https://doi.org/10.1136/thx.2003.016667
16. Ferrante G, Antona R, Malizia V, Montalbano L, Corsello G, La Grutta S. Smoke exposure as a risk factor for asthma in childhood: a review of current evidence. Allergy Asthma Pro 2014;35:454-61. https://doi.org/10.2500/aap.2014.35.3789

17. National Center for Chronic Disease Prevention and Health Promotion (US) Office on Smoking and Health. The health consequences of smoking: 50 years of progress. A report of the surgeon general. Centers for Disease Control and Prevention (US) Atlanta, 2014.

18. Burke $\mathrm{H}$, Leonardi-Bee J, Hashim A, Pine-Abata $\mathrm{H}$, Chen $Y$, Cook DG, et al. Prenatal and passive smoke exposure and incidence of asthma and wheeze: systematic review and meta-analysis. Pediatrics 2012;129:735-44. https://doi.org/10. 1542/peds.2011-2196

19. Saulyte J, Regueira C, Montes-Martínez A, Khudyakov P, Takkouche B. Active or passive exposure to tobacco smoking and allergic rhinitis, allergic dermatitis, and food allergy in adults and children: a systematic review and meta-analysis. PLoS Med 2014;11:e1001611. https://doi.org/10.1371/journal.pmed.1001611

20. Thacher JD, Gruzieva O, Pershagen G, Neuman $\AA$, van Hage M, Wickman M, et al. Parental smoking and development of allergic sensitization from birth to adolescence. Allergy 2016;7:239-48. https://doi.org/10.1111/all.12792

21. Rusconi F, Galassi C, Bellasio M, S. Piffer, E. Lombardi, E. Bonci, et al; Gruppo Collaborativo SIDRIA-2. Risk factors in the pre-, perinatal and early life (first year) for wheezing in young children. Epidemiol Prev 2005;29:47-51.

22. Kukla L, Hrubá D, Tyrlik M. Trends in respiratory morbidity of children in relation to their passive smoking exposure. Cent Eur J Public Health 2006;14:180-5. https://doi. org/10.21101/cejph.a3386

23. Vork KL, Broadwin RL, Blisdell RJ. Developing asthma in childhood from exposure to secondhand tobacco smoke: insight from meta-regression. Environ Health Perspect 2007;115:1394-400. https://doi.org/10.1289/ehp.10155

24. Cibella F, Cuttitta G, La Grutta S, Melis MR, Lospalluti $M L$, Uasuf CG, et al. Proportional Venn diagram and determinants of allergic respiratory diseases in Italian adolescents. Pediatr Allergy Immunol 2011;22:60-8. https://doi.org/10.1111/j.1399-3038.2010.01097.x

25. De Sario M, Forastiere F, Viegi G, Simoni M, Chellini E, Piccioni $\mathrm{P}$, et al; Gruppo Collaborativo SIDRIA-2. Fumo dei genitori e disturbi respiratori infantili. Epidemiol Prev 2005;29:52-6.

26. Ferrante G, Simoni M, Cibella F, Ferrara F, Liotta G, Malizia V,et al. Third-hand smoke exposure and health hazards in children. Monaldi Arch Chest Dis 2013;79:38-43. https:// doi.org/10.4081/monaldi.2013.108

27. Mahabee-Gittens EM, Merianos AL, Matt GE. Preliminary evidence that high levels of nicotine on children's hands may contribute to overall tobacco smoke exposure. Tob Control 2018;27:217-9. https://doi.org/10.1136/tobaccocontrol-2016-053602

28. Tung $\mathrm{KY}$, Wu KY, Tsai $\mathrm{CH}$, Su MW, Chen $\mathrm{CH}$, Lin $\mathrm{MH}$, et al. Association of time-location patterns with urinary cotinine among asthmatic children under household environmental tobacco smoke exposure. Environ Res 2013;124:7-12. https://doi.org/10.1016/j.envres.2013.03.002

29. Damera G, Panettieri RA. Irreversible airway obstruction in asthma: what we lose, we lose early. Allergy Asthma Proc 2014;35:111-8. https://doi.org/10.2500/aap.2013.34.3724 
30. Jung JW, Ju YS, Kang HR. Association between parental smoking behavior and children's respiratory morbidity: 5 -year study in an urban city of South Korea. Pediatr Pulmonol 2012;47:338-45. https://doi.org/10.1002/ppul.21556

31. Vardavas $\mathrm{Cl}$, Filippidis FT, Agaku IT. Determinants and prevalence of e-cigarette use throughout the European Union: a secondary analysis of 26566 youth and adults from 27 Countries. Tob Control 2015;24:442-8. https://doi. org/10.1136/tobaccocontrol-2013-051394

32. Wang MP, Ho SY, Leung LT, Lam TH. Electronic cigarette use and its association with smoking in Hong Kong Chinese adolescents. Addict Behav 2015;50:124-7. https://doi.org/10.1016/j.addbeh.2015.06.037

33. Cooke A, Fergeson J, Bulkhi A, Casale TB. The electronic cigarette: the good, the bad, and the ugly. J Allergy Clin Immunol Pract 2015;3:498-505. https://doi.org/10.1016/j.jaip.2015.05.022

34. Lødrup Carlsen KC, Skjerven HO, Carlsen KH. The toxicity of E-cigarettes and children's respiratory health. Paediatr Respir Rev 2018;28:63-7. https://doi.org/10.1016/j.prrv.2018.01.002

35. Clapp PW, Jaspers I. Electronic cigarettes: their constituents and potential links to asthma. Curr Allergy Asthma Rep 2017;17:79. https://doi.org/10.1007/s11882-017-0747-5

36. Ferkol TW, Farber HJ, La Grutta S, Leone FT, Marshall HM, Neptune E, et al.; Forum of International Respiratory Societies. Electronic cigarette use in youths: a position statement of the Forum of International Respiratory Societies. Eur Respir J 2018;51:1800278. https://doi.org/10.1183/ 13993003.00278-2018

37. Trehy HL, Ye W, Hadwiger ME, Moore TW, Allgire JF, Woodruff JT, et al. Analysis of electronic cigarette cartridges, refill solutions, and smoke for nicotine and nicotine related impurities. J Liq Chromatogr Relat Technol 2011;34:144258. https://doi.org/10.1080/10826076.2011.572213

38. Food and Drug Administration, HHS. Deeming tobacco products to be subject to the federal food, drug, and cosmetic act, as amended by the family smoking prevention and tobacco control act; regulations on the sale and distribution of tobacco products and required warning statements for tobacco products; proposed rule. Federal Register 2014;79:23142-207.

39. Leigh NJ, Lawton RI, Hershberger PA, Goniewicz M. Flavourings significantly affect inhalation toxicity of aerosol generated from electronic nicotine delivery systems (ENDS). Tob Control 2016;25:ii81-7. https://doi.org/10.1136/tobaccocontrol-2016-053205

40. Dinakar C, O'Connor GT. The health effects of electronic cigarettes. N Engl J Med 2016;375:1372-81. https://doi. org/10.1056/NEJMra1502466

41. Wang MP, Ho SY, Leung LT, Lam TH. Electronic cigarette use and respiratory symptoms in Chinese adolescents in Hong Kong. JAMA Pediatr 2016;170:89-91. https://doi.org/ 10.1001/jamapediatrics.2015.3024

42. Singh T, Agaku IT, Arrazola RA, Marynak KL, Neff LJ, Rolle IT, et al. Exposure to advertisements and electronic ci- garette use among US middle and high school students. Pediatrics 2016;137:e20154155. https://doi.org/10.1542/ peds.2015-4155

43. Bunnell RE, Agaku IT, Arrazola RA, Apelberg BJ, Caraballo RS, Corey CG, et al. Intentions to smoke cigarettes among never-smoking US middle and high school electronic cigarette users: National Youth Tobacco Survey, 20112013. Nicotine Tob Res 2015;17:228-35. https://doi.org/ 10.1093/ntr/ntu166

44. Coleman BN, Apelberg BJ, Ambrose BK, Green KM, Choiniere CJ, Bunnell R, et al. Association between electronic cigarette use and openness to cigarette smoking among US young adults. Nicotine Tob Res 2015;17:212-8. https:// doi.org/10.1093/ntr/ntu211

45. Ghosh A, Coakley RC, Mascenik T, Rowell TR, Davis ES, Rogers K, et al. Chronic e-cigarette exposure alters the human bronchial epithelial proteome. Am J Respir Crit Care Med 2018;198:67-76. https://doi.org/10.1164/rccm.201710$20330 \mathrm{C}$

46. Smit LA, Bouzigon E, Pin I, Siroux V, Monier F, Aschard $\mathrm{H}$, et al.; EGEA Cooperative Group. 17q21 variants modify the association between early respiratory infections and asthma. Eur Respir J 2010;36:57-64. https://doi.org/10.1183/ 09031936.00154509

47. Thacher JD, Gehring U, Gruzieva O, Standl M, Pershagen $\mathrm{G}$, Bauer $\mathrm{CP}$, et al. Maternal smoking during pregnancy and early childhood and development of asthma and rhinoconjunctivitis - a MeDALL Project. Environ Health Perspect 2018;126:047005. https://doi.org/10.1289/EHP2738

48. Silvestri M, Franchi S, Pistorio A, Petecchia L, Rusconi F. Smoke exposure, wheezing, and asthma development: a systematic review and meta-analysis in unselected birth cohorts. Pediatr Pulmonol 2015;50:353-62. https://doi.org/ 10.1002/ppul.23037

49. Carroll KN, Gebretsadik T, Griffin MR, Dupont WD, Mitchel EF, Wu P, et al. Maternal asthma and maternal smoking are associated with increased risk of bronchiolitis during infancy. Pediatrics 2007;119:1104-12. https://doi. org/10.1542/peds.2006-2837

50. Ministero della Salute. Conferenza permanente per i rapporti tra lo Stato le Regioni e le Province autonome di Trento e Bolzano. Gazzetta Ufficiale n. 276 del 17/09/2001.

51. Gerald LB, Gerald JK, Gibson L, Patel K, Zhang S, McClure LA. Changes in environmental tobacco smoke exposure and asthma morbidity among urban school children. Chest 2009;13:911-6. https://doi.org/10.1378/chest.08-1869

52. Nurmagambetov TA, Barnett SB, Jacob V, Chattopadhyay SK, Hopkins DP, Crocker DD, et all. Task Force on Community Preventive Services. Economic value of home-based, multi-trigger, multicomponent interventions with an environmental focus for reducing asthma morbidity a community guide systematic review. Am J Prev Med 2011;41:S33-47. https:// doi.org/10.1016/j.amepre.2011.05.011

53. GARD Italy. La tutela dal fumo passivo negli spazi confinati o aperti non regolamentati dalla Legge 3/2003 art.51 (Legge Sirchia) e successive modificazioni. Giugno 2017. 